\title{
Seminal Vesicle Infection of Zinner Syndrome Misdiagnosed for Neoplasm
}

\author{
Young Joo Kim \\ Department of Urology, Jeju National University School of Medicine, Jeju, Korea
}

Zinner syndrome is a rare Müllerian duct congenital anomaly representing unilateral renal agenesis, ipsilateral seminal vesicle cyst, and ejaculatory duct obstruction. A 16-year-patient presented with dysuria, nocturia, and enduring diffuse perineal pain for two months. Radiologic studies revealed a right seminal vesicle cyst with a mass lesion and right renal agenesis. Ultrasonography guided transrectal biopsy indicated a seminal vesicle neoplasm. The mass was diagnosed as chronic inflammation. This paper reports a case of seminal vesicle inflammation associated with Zinner syndrome that was initially misdiagnosed as a neoplasm.

Keywords: Solitary kidney; Seminal vesicle; Neoplasm; Infections

Copyright $\odot$ 2020, Korean Association of Urogenital Tract Infection and Inflammation. All rights reserved. (c) (1) (\$) This is an open access article distributed under the terms of the Creative Commons Attribution (c) (1) Non-Commercial License (http://creativecommons.org/licenses/by-nc/4.0) which permits unrestricted non-commercial use, distribution, and reproduction in any medium, provided the original work is properly cited.
Received: 10 July, 2020

Revised: 13 August, 2020

Accepted: 15 August, 2020
Zinner syndrome is a very rare congenital anomaly that is characterized by features of ipsilateral renal agenesis, seminal vesicle cyst, and ejaculatory duct obstruction [1]. The related symptoms showed mainly nonspecific urologic symptoms, such as lower urinary tract symptoms, painful voiding, abnormal ejaculation, and diffuse perineal ache [2]. Most patients are diagnosed after post-adolescence owing to the insufficient symptoms. This paper reports a 16-year-old male with a seminal vesicle infection of Zinner syndrome that was misdiagnosed as a neoplasm. The study was approved by the Institutional Review Board of the Jeju National University Hospital (IRB no. JEJUNUH 2020-04027).

\section{CASE REPORT}

A 16-year-old patient presented with dysuria, nocturia, and diffuse perineal pain for two months. The patient had a history of acute epididymitis four years earlier. Urinalysis showed no abnormalities, and he had been diagnosed with renal agenesis. Therefore, radiologic studies were con- ducted. Computed tomography showed a solitary left kidney (Fig. 1A). Ultrasonography (US) of the prostate revealed a hetero-echoic mass in several enlarged seminal vesicle cysts (Fig. 1B).

A Tc-99m dimercaptosuccinic acid renal scan was performed to identify the invisible right kidney and evaluate the seminal vesicle mass. The right kidney did not accumulate radionuclide and was not visible (Fig. 1C). Magnetic resonance imaging (MRI) was performed. On MRI, a seminal vesicle tumor appeared as a heterogeneous mass with an intermediate signal on the T1-weighted image (WI) and as heterogeneous high signal intensity on the T2-WI (Fig. 1D). The radiology findings revealed a solitary left kidney and several large seminal vesicle cysts with a $3 \times 1.7 \mathrm{~cm}$ sized tumor lesion. No abnormalities were found in the abdominal and external genital physical examination. A digital rectal examination detected a no palpable lesion of the seminal vesicle and a normal-sized prostate.

The level of prostate-specific antigen was $0.27 \mathrm{ng} / \mathrm{ml}$. Follicle-stimulating hormone, luteinizing hormone, and testosterone were within the normal ranges. The patient was 

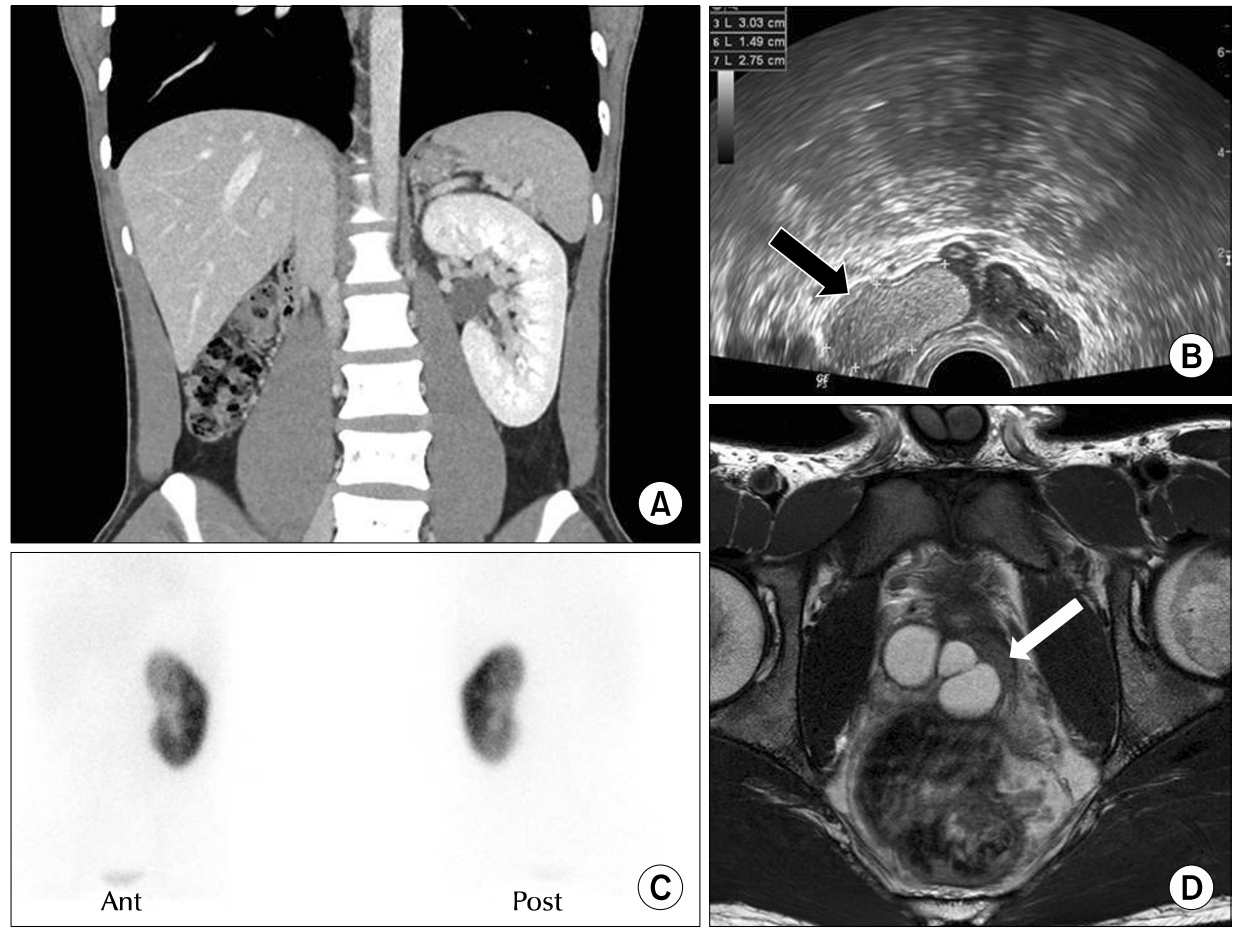

Fig. 1. (A) Computed tomography shows a solitary left kidney. (B) Prostate ultrasound shows the cystic tubular structure and $3 \times 1.7 \mathrm{~cm}$ sized tumor lesion with internal echo (black arrow). (C) Tc-99m dimercaptosuccinic acid renal scan. The right kidney did not accumulate radionuclide and as not visible along the whole study. (D) Axial T2W magnetic resonance image show multiple cystic hyper-intense structures with intermediate signal intensity mass lesion (white arrow). Ant: anterior, Post: poterior. suspected of Zinner syndrome with a seminal vesicle neoplasm. A US-guided trans-rectal needle biopsy was performed to diagnose a pathological result. A biopsy of the seminal vesicle tumor was negative for malignancy. The histopathological examination revealed chronic inflammatory cells. The author treated the patient with conservative medical methods. Therefore, antibiotics were prescribed for seven days (ciprofloxacin $500 \mathrm{mg}$ twice), but his complaints were not resolved. The result of culture showed 'no growth of organism'. Therefore, additional analgesic and ciprofloxacin were prescribed for 14 days, and the symptoms improved completely. He was followed up for six months after treatment and had no complaints. The US finding showed a dilated cystic complex of the seminal vesicle, but no previous solid lesion.

\section{DISCUSSION}

Zinner syndrome is defined by ipsilateral renal agenesis, seminal vesicle cyst, and ipsilateral ejaculatory duct obstruction. The prevalence of unilateral renal agenesis is $0.1 \%$ of newborns [2], and the relevant embryologic associations between the genitourinary tracts describe the developmental deviations, leading to abnormality. Dysfunctional migration of the ureteric bud indicates a failure to join with the metanephros. This is likely to hinder the ureteric bud as the main cause in the differentiation of the metanephric blastemal, and ipsilateral renal agenesis will be generated that will block the ejaculatory duct [3]. The cause of the result was inadequate flow and subsequent expansion of the seminal vesicle indicating a cystic structure [4]. The patients are usually diagnosed by presenting with urologic symptoms, such as painful voiding, frequent voiding, perineal pain, and epididymitis after the post-adolescent years [5]. In the present study, the patient presented with dysuria, nocturia, and perineal pain. Small cysts may have no symptoms, whereas large cysts usually cause bladder irritation and obstruction. The patients are also diagnosed with infertility due to oligospermia and azoospermia in the middle years of one's life [6]. Imaging can assist in an accurate diagnosis of Zinner syndrome. If patients have symptoms, an exact diagnosis of radiologists can help a urologist make a therapeutic decision. US can help find ipsilateral renal agenesis, and the obstructed ejaculatory ducts are shown as echo-free mixtures in the pelvis [7]. US may provide high accuracy imaging in many cases, but there may be difficulties in obese patients. Both abdominopelvic and trans-rectal US are recommended to identify the adjacent anatomical structures surrounding the several seminal vesicle cysts. MRI is the modality of choice for exhibiting seminal vesicles and evaluating associated anomalies. In addition, it can help differentiate the seminal 
vesicle cysts from other pelvic masses [8]. Treatment should be related to subjective clinical manifestation. Surveillance is a useful modality in the absence of clinical symptoms.

The surgical approach may be considered if conservative options are ineffective. An evaluation of sperm through US-guided trans-rectal aspiration of a cyst can also confirm Zinner syndrome. Considerable therapeutic modalities for the symptomatic relief are cystic aspiration, laparoscopic guided drainage, and transurethral removal of cystic wall. An ejaculatory ductal obstruction causes infertility, which can be reestablished when the obstruction is released. Some studies reported that trans-rectal aspiration may temporarily relieve symptoms and may be helpful in diagnosis through the instillation of contrast dye [9].

On the other hand, cyst aspiration is not recommended, mainly because the cure rate of treatment is approximately $30 \%$, and there is a noticeable risk of infection. Antibiotics with analgesics were prescribed, and the patient remained asymptomatic. Less than 60 cases of tumors from the seminal vesicle have been reported, and cases of malignancy-related to congenital malformation of the urogenital system are sporadic. Therefore, there are no recommendations for detecting the hidden malignancy of seminal vesicle, but a seminal vesicular mass biopsy is recommended, as in this case.

\section{CONFLICT OF INTEREST}

No potential conflict of interest relevant to this article was reported.

\section{ACKNOWLEDGMENTS}

This work was supported by the 2020 education, research and student guidance grant funded by Jeju National University.

\section{REFERENCES}

1. Humphrey PA. Clear cell neoplasms of the urinary tract and male reproductive system. Semin Diagn Pathol 1997;14: 240-52.

2. Florim S, Oliveira V, Rocha D. Zinner syndrome presenting with intermittent scrotal pain in a young man. Radiol Case Rep 2018;13:1224-7.

3. Nassir A. Symptomatic cystic seminal vesicle: a laparoscopic approach for effective treatment. Can Urol Assoc J 2009;3: E81-3.

4. Juho YC, Wu ST, Tang SH, Cha TL, Meng E. An unexpected clinical feature of Zinner's syndrome - a case report. Urol Case Rep 2015;3:149-51.

5. Jarzemski P, Listopadzki S, Kowalski M. Laparoscopic removal of a congenital seminal vesicle cyst in Zinner's syndrome. JSLS 2014;18:367-71.

6. Ghonge NP, Aggarwal B, Sahu AK. Zinner syndrome: a unique triad of mesonephric duct abnormalities as an unusual cause of urinary symptoms in late adolescence. Indian J Urol 2010;26: 444-7.

7. Jiang XS, Wang HJ, Lin JH, Guo Y, Sun CH, Lin L, et al. Zinner's syndrome: clinical features and imaging diagnosis. Asian J Androl 2018;20:316-7.

8. Mehra S, Ranjan R, Garga UC. Zinner syndrome-a rare developmental anomaly of the mesonephric duct diagnosed on magnetic resonance imaging. Radiol Case Rep 2016;11:313-7.

9. Fiaschetti V, Greco L, Giuricin V, De Vivo D, Di Caprera E, Di Trapano $\mathrm{R}$, et al. Zinner syndrome diagnosed by magnetic resonance imaging and computed tomography: role of imaging to identify and evaluate the uncommon variation in development of the male genital tract. Radiol Case Rep 2016;12:54-8. 